\title{
Myocardial late gadolinium enhancement cardiovascular magnetic resonance in patients with cirrhosis
}

\author{
Dirk Lossnitzer ${ }^{1}$, Henning Steen ${ }^{1}$, Alexandra Zahn², Stephanie Lehrke ${ }^{1}$, Celine Weiss' ${ }^{1}$ Karl Heinz Weiss², \\ Evangelos Giannitsis ${ }^{1}$, Wolfgang Stremmel ${ }^{2}$, Peter Sauer ${ }^{2}$, Hugo A Katus ${ }^{1}$, Daniel N Gotthardt ${ }^{2 *}$
}

\begin{abstract}
Background: Portal hypertension and cardiac alterations previously described as "cirrhotic cardiomyopathy" are known complications of end stage liver disease (ELD). Cardiac failure contributes to morbidity and mortality, particularly after liver transplantation and transjugular intrahepatic portosystemic shunt (TIPS). We sought to identify myocardial tissue characterization and evaluate cardiovascular magnetic resonance (CMR) for diagnosis of cardiac impairment.

Results: Twenty ELD patients underwent CMR for morphological, functional and tissue characterization by late gadolinium enhancement (LGE). Based on extent of LGE, patients were dichotomized into high and low LGE groups and analyzed regarding liver, cardiocirculatory and renal functions. CMR demonstrated hyperdynamic left ventricular function and a patchy pattern of LGE of the myocardium to a variable extent (range 2-62\%) in all patients. There were no significant differences in Model for End-Stage Liver Disease (MELD), Child-Pugh score or the left ventricular ejection fraction between high and low LGE groups. QTc-interval was prolonged in 25\% of the patients. E/A ratio was at the upper limit of norm; no difference between groups. Patients showing high LGE had a higher $\mathrm{Cl}(\mathrm{p}<0.05)$. Biomarkers of myocardial stress were elevated. While NT-proBNP and c-Troponin-T showed no differences, PLGF and sFLT1 were lower in the high LGE group.

Conclusion: CMR shows myocardial involvement in patients with ELD resembling appearance of myocarditis. The hyperdynamic circulation in portal hypertension may be an important factor. Larger prospective trials are warranted to confirm the association with severity and outcome of liver disease and to test the predictive power of CMR for patients listed for liver transplantation.
\end{abstract}

\section{Background}

End stage liver disease (ELD) regularly leads to major alterations in the regulation of the cardiovascular system. Portal hypertension and/or hormonal changes in ELD induce a hyperdynamic circulatory state characterized by arterial hypotension and tachycardia and are often accompanied by ascites and electrolyte disturbances. Recent data also emphasizes the impact of liver function on renal (hepatorenal syndrome) and pulmonary (hepatopulmonary and portopulmonary syndrome) circulation [1], [2]. Driven by the high morbidity and

\footnotetext{
*Correspondence: daniel_gotthardt@med.uni-heidelberg.de

${ }^{2}$ Department of Internal Medicine IV, University Hospital of Heidelberg, Im

Neuenheimer Feld 410, 69120 Heidelberg, Germany

Full list of author information is available at the end of the article
}

mortality, significant research has been devoted to the causes and treatment of renal and pulmonary manifestations. Contrary to these efforts, little data is available on myocardial changes due to ELD. Although the hypothesis of ethanol-induced cardiomyopathy has been around for several decades, the role of the heart in the hypercirculatory state itself has only been addressed in a small number of studies [3], [4], [5]. Apparently, the hypercirculatory state was considered as proof of normal contractile function of the heart. A decreased ejection fraction is found only in a minority of cases with ELD. Some researchers propagate a specific disease entity called "cirrhotic cardiomyopathy" for the cases of advanced reduction of ejection fraction and for more subtle evidences of functional impairment such as
C Biomed Central

(c) 2010 Lossnitzer et al; licensee BioMed Central Ltd. This is an Open Access article distributed under the terms of the Creative Commons Attribution License (http://creativecommons.org/licenses/by/2.0), which permits unrestricted use, distribution, and reproduction in any medium, provided the original work is properly cited. 
prolonged QTc-interval, impaired response to physical activity/stress, reduced heart rate variability, or altered $\mathrm{E} / \mathrm{A}$ in echocardiography eventually resulting in higher morbidity and mortality pre- and post-OLT[6], [3]. The improved survival of patients with ELD after orthotopic liver transplantation (OLT) and the hypercirculatory state after OLT and transjugular intrahepatic portosystemic shunt (TIPS) have directed research interests towards the myocardium [7], [8], [9].

Cardiovascular magnetic resonance (CMR) has become the gold standard method for the assessment of cardiac morphology and function in various cardiomyopathies (CMP) [10]. Areas of high signal intensity appearing 10-15 min after injection of the intercellular contrast agent gadolinium were first described as late enhancement (LGE) in regions of myocardial scarring after myocardial infarction [11]. This technique allows an additional, excellent imaging modality for the analysis of intercellular matrix. LGE can be detected in postinfarct scars, inflammation, as well as non-ischemic CMP, cardiac neoplasm and storage diseases such as amyloidosis. Typical LGE patterns have been defined for each of these diseases [12]. Moreover the extent of LGE is associated with the amount of affected myocardial tissue, e.g. in myocarditis or ischemic cardiomyopathy [13]. CMR is the preferred test for the repeated evaluation of disease course due to the lack of radiation and its non-invasive character [14].

Here we used CMR and serological markers for the evaluation of functional myocardial changes, non-invasive tissue characterization and the identification of specific cardiac lesions in cirrhotic patients listed for liver transplantation.

\section{Methods}

\section{Patients}

From March 2007 until July 2008, 20 consecutive patients with ELD had a CMR scan at the department of cardiology of the University of Heidelberg, Germany. All patients had been referred by the department of gastroenterology of the University of Heidelberg, Germany for evaluation of cardiac functional analysis. Exclusion criteria were decreased kidney function (glomerular filtration rate below $30 \mathrm{ml} / \mathrm{min} / 1.73 \mathrm{~m}^{2}$ ) and general contraindications to CMR (e.g., metal implants, pacemaker, and claustrophobia). All procedures used in this study complied with the Declaration of Helsinki, were approved by our local ethics committee, and all patients gave written informed consent.

All patients were listed for liver transplantation. Two were waiting for re-transplantation due to chronic transplantation failure. Clinical characteristics are shown in Table 1 . The mean age was $52.4 \pm 9.4$ years. The etiology of cirrhosis was alcohol in 12 patients, virus
Table 1 Patient characteristics

\begin{tabular}{|c|c|c|}
\hline Parameter $(n=20)$ & $\begin{array}{l}\text { Cirrhosis } \\
\text { patients }\end{array}$ & Controls \\
\hline Age (mean \pm SD) [years] & $52.4 \pm 9.4(24-68)$ & $\begin{array}{l}42.0 \pm 12.9(24- \\
69)\end{array}$ \\
\hline $\begin{array}{l}\text { Body mass index (mean } \pm \text { SD) } \\
{\left[\mathrm{kg} / \mathrm{m}^{2}\right]}\end{array}$ & $\begin{array}{l}25.6 \pm 4.9(16.9- \\
34.0)\end{array}$ & $\begin{array}{l}24.2 \pm 3.3(18.9- \\
33.0)\end{array}$ \\
\hline $\operatorname{Sex}(M / F)$ & $11 / 9$ & $60 / 60$ \\
\hline Bilirubin (mean \pm SD) [mg/dL] & $\begin{array}{l}4.68 \pm 6.7(0.33- \\
28.8)\end{array}$ & N/A \\
\hline Albumin (mean $\pm \mathrm{SD}$ ) [g/L] & $\begin{array}{l}30.34 \pm 7.2(19- \\
46)\end{array}$ & N/A \\
\hline MELD score (mean \pm SD) & $\begin{array}{l}14.9 \pm 6.3(6.4- \\
28.5)\end{array}$ & $\mathrm{N} / \mathrm{A}$ \\
\hline Child-Pugh score (median) & $9(5-11)$ & $\mathrm{N} / \mathrm{A}$ \\
\hline \multicolumn{3}{|l|}{ Etiology } \\
\hline Alcoholic & 12 & \\
\hline Non-alcoholic & 8 & \\
\hline Autoimmune & 3 & \\
\hline Viral & 3 & \\
\hline Cryptogenic & 2 & \\
\hline
\end{tabular}

MELD score Model for End-Stage Liver Disease; Range is in brackets, SD standard deviation, N/A not assessed

infection in three, autoimmune disease in three, and cryptogenic in two patients, no patient suffered from malignant disease. Gender distribution was 11 male and 9 female. The median Child-Pugh score was 9 (range 5-11) and the mean Model for End-Stage Liver Disease (MELD) score was $14.9 \pm 6.3$. No patient revealed clinical signs of impaired left ventricular dysfunction except one who had been diagnosed as "alcoholic cardiomyopathy" and showed moderately impaired left ventricular function. All patients were on therapy with non-selective beta-blockers (Propanolol).

Routine echocardiography was performed on a Philips ie33 echo system (Philips Medical Systems, Netherlands). Doppler ratio of early to late transmitral flow velocity (E/A) was calculated.

\section{Healthy reference group}

The hemodynamics of ELD patients were compared with a healthy reference group of 120 volunteers within our center. Subjects were considered as healthy (mean age was $42.0 \pm 12.9$ years) when no severe diseases were found in their medical history and a clinical examination, routine blood parameters, ECG, blood pressure measurements, spirometry as well as dobutamine stress and LGE CMR were within a normal range..

\section{CMR}

All images were acquired with a $1.5 \mathrm{~T}$ magnetic resonance imaging system (ACHIEVA, Philips Medical Systems, Netherlands), a flexible cardiac five-element 
phased array coil and a vector electrocardiogram for $\mathrm{R}$ wave triggering using a standard MRI imaging protocol.

In brief, multiple short axis (SAX) cine images using a steady state free precession sequence with parallel imaging (balanced Fast Field Echo (FFE); Repetition time $(\mathrm{TR}) /$ Echo time $(\mathrm{TE})=2.9 / 1.45 \mathrm{~ms}$; reconstructed voxel-size $=1.5 \times 1.5 \times 8 \mathrm{~mm}$ acquisition; sensitivity encoding (SENSE)-factor $=2$ ) were acquired after the acquisition of true two- and four-chamber planes for the assessment of left ventricular ejection fraction (LVEF \%).

A 2D FFE multi-slice SAX inversion recovery sequence $(\mathrm{TR} / \mathrm{TE}=2.9 / 1.0$; reconstructed voxel-size $=$ $2.0 \times 2.0 \times 5 \mathrm{~mm}$ acquisition; SENSE-factor $=2$; $\mathrm{TI}=$ $200-280 \mathrm{~ms}) 10$ minutes post-Gd $(\mathrm{Gd}=0.2 \mathrm{mmol} / \mathrm{kg}$ of gadopentetate dimeglumine (Schering, Berlin, Germany)) was employed for the measurement of LGE.

The scan protocol was carried out in the following order: after a survey and reference scan, balanced cine FFE were acquired in 2-chamber, 4-chamber, short axis (multi-slice), and 3-chamber orientations. LGE scans were carried out in SAX, 4-chamber, and 2-chamber orientations 10 minutes after the injection of gadolinium. The inversion time was chosen very carefully by performing a T1 mapping using a lock locker sequence in each patient to estimate the correct inversion time (Fig. 1). The evidence of LGE was confirmed by imaging different views of the myocardium (i.e. 4 chamber view, 2 chamber view as well as short axis views).

\section{Image analysis}

All CMR images were analyzed on a commercially available Cardiovascular Magnetic Resonance workstation with standard software (Philips Viewforum, Version 5.0,

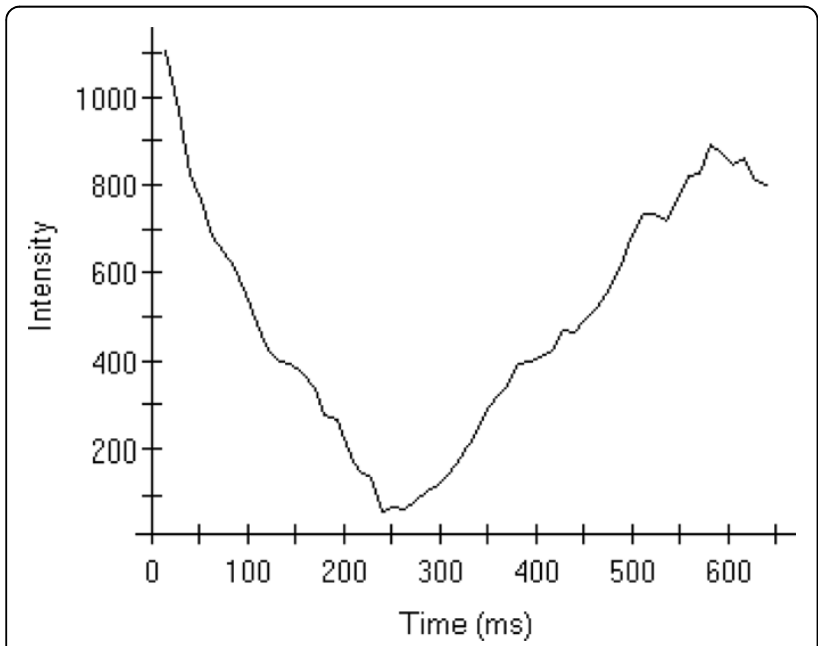

Figure 1 T1 Mapping intensity graph based on a lock locker sequence to quantify the optimal inversion time for LGE imaging
Best, Netherlands) by consensus reading of two blinded experienced observers.

\section{Definition of global LV function}

For cine imaging, a modified 16-segment model according to the AHA definition [15] for the left ventricle was used to analyze left ventricular function and delayed enhancement per segment.

LV global function were calculated by the Philips View Forum software, version 5.0, after the manual tracing of the endocardial contours of the left ventricular wall at end diastole and end systole on the short axis cine data set.

\section{Analysis of late gadolinium enhancement}

Segmental enhancement was quantified by the Philips View Forum Software 5.0 in all 16 segments and declared as a percentage of LGE of LV mass (Fig. 2). Signal intensity was measured as mean signal intensity plus standard deviations of mean (SD) for intra-individual analysis. Enhancement was defined as a signal intensity greater than five SD above the acquired mean signal intensity level [16].

To evaluate the association of late enhancement with the indices of cardiovascular stress or with indices of liver disorders, we dichotomized the patients in two equally large groups in respect to the percentage of myocardium with late enhancement.

\section{Clinical scores and blood tests}

Blood sampling was carried out on the same day of the MRI scan. Blood samples were sent to the central laboratory of the University Hospital Heidelberg for routine parameters. Plasma aliquots were frozen at $-80^{\circ} \mathrm{C}$ until biomarker assays were performed. High sensitivity Troponin T (hsTNT) was measured with a pre-commercial assay by electrochemo-luminescence methods (Roche Diagnostics, Mannheim, Germany).

Cardiac Troponin T (cTNT) was measured using the $4^{\text {th }}$ generation commercial one-step electrochemiluminescence assay based on electrochemoluminescence technology (Elecsys 2010, Roche Diagnostics, Mannheim, Germany).

$\mathrm{N}$-terminal prohormone brain natriuretic peptide (NT-proBNP) was measured using a highly sensitive and specific electrochemluminescence immunoassay (Elecsys proBNP, Roche Diagnostics, Mannheim, Germany). The measurement range extends from 5 to $35,000 \mathrm{pg} / \mathrm{mL}$. The minimal detectable concentration is $5 \mathrm{pg} / \mathrm{mL}$ and the coefficient of variation is $5.7 \%$ at $64 \mathrm{pg} / \mathrm{mL}$.

PLGF and sFLT1 were measured using Elecsys ${ }^{\oplus}$ PLGF (measurement range extends from 3 to $10,000 \mathrm{pg} / \mathrm{mL}$ ) und Elecsys ${ }^{\oplus}$ sFLT1 (measurement range extends from 10 to $85,000 \mathrm{pg} / \mathrm{mL}$ ) routine assays by Roche Diagnostics, Mannheim, Germany. 


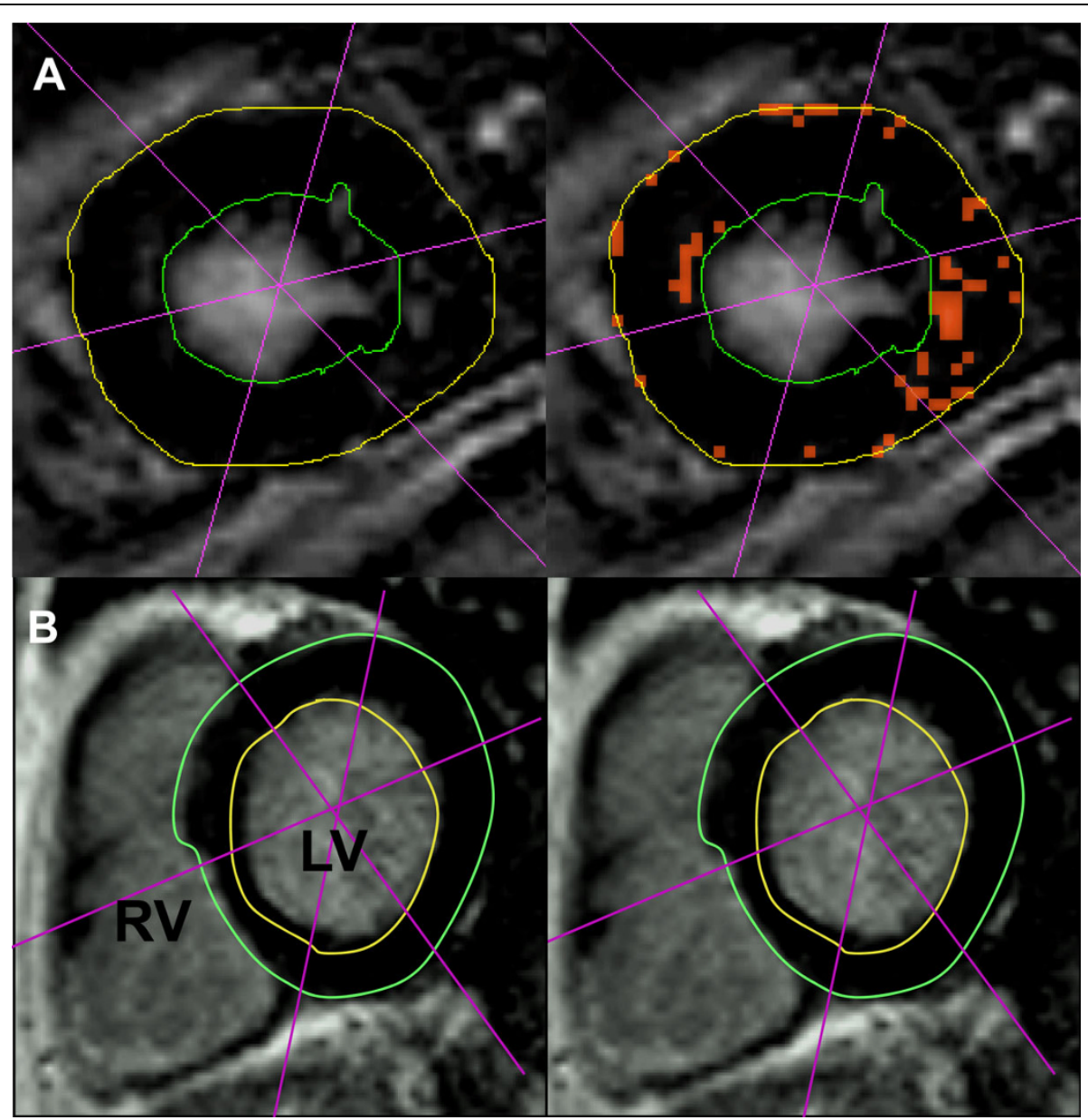

Figure 2 LGE quantification in short-axis orientation in ELD patients with low LGE (detected by orange dots, $11 \%$ of myocardial mass (A) and a normal subject without delayed enhancement (B). $L V=$ left ventricle, $R V=$ right ventricle, $S=$ interventricular septum, ${ }^{*} p<0.05$

\section{Statistical analysis}

All statistical analyses were performed using SPSS 16 (SPSS Inc. Chicago, Illinois). Parametric (t tests) and non-parametric (Mann-Whitney $U$ test) tests were used for continuous data. In addition, the Fisher exact test was used where applicable. All $\mathrm{p}<0.05$ were considered statistically significant.

\section{Results}

\section{Evidence of hyperdynamic circulation in ELD patients}

Hemodynamic parameters measured by CMR of the patients are shown in Table 2 and were then compared to data of the healthy reference group. Values are given as mean and $+/$ - standard deviation: Heart rate was $74.9 \pm 11.9 / \mathrm{min}$ (reference group $64.8 \pm 9.4 / \mathrm{min}$, $\mathrm{p}<0.01$ ) and stroke volume $107 \pm 20.8 \mathrm{~mL}$ (reference group $91.7 \pm 30.3 \mathrm{~mL}, \mathrm{p}=0.08)$. Left ventricular ejection fraction (LVEF) was $72 \pm 7.5 \%$ with a range from $49 \%$ to $83 \%$ (reference group $65.8 \pm 6.96 \%$ with a range from
$32.8 \%$ to $84 \%, \mathrm{p}<0.01)$. Cardiac output was $7.9 \pm 1.63$ $\mathrm{L} / \mathrm{min}$ with a range from 4.4 to $11.1 \mathrm{~L} / \mathrm{min}$ (reference group $6.7 \pm 1.6 \mathrm{~L} / \mathrm{min}$ with a range from 3.5 to 12.5 , $\mathrm{p}<0.01)$. The resulting CI yielded $4.36 \pm 0.86 \mathrm{~L} / \mathrm{min} / \mathrm{m}^{2}$, ranging from 3.41 to $6.48 \mathrm{~L} / \mathrm{min} / \mathrm{m}^{2}$ (reference group $3.6 \pm 0.7 \mathrm{~L} / \mathrm{min} / \mathrm{m}^{2}$, ranging from 2.1 to $6.3 \mathrm{~L} / \mathrm{min} / \mathrm{m}^{2}$, $\mathrm{p}<0.001)$. Left-ventricular end-diastolic volume was $150.0 \pm 35.4 \mathrm{~mL}$ (reference group 149.4 $\pm 33.8 \mathrm{~mL}$, $\mathrm{p}=0.91)$. These changes were in line with previous observations of hyperdynamic circulation as part of the altered cardiovascular regulation in patients with ELD.

\section{LGE CMR}

We found variable degrees of LGE in all patients examined, indicative of myocardial injury despite hypernormal circulatory indices (Fig. 3). The amount of LGE measured in percentage of myocardium revealed a mean of $27 \% \pm 16.5 \%$ and showed a patchy pattern similar to patients with myocarditis [17]. The minimum amount 
was $2 \%$, the maximum as much as $62 \%$ (Table 2), the median was $25 \%$. The detection of these changes was independent of the etiology of liver disease and was observed in patients with alcohol-induced, virus-related, autoimmune or cholestatic hepatic disease. No LGE was found in the reference group.

\section{Relationship of LGE with clinical indices of liver disease}

We dichotomized the patients regarding the percentage of myocardium with late enhancement resulting in a high LGE group and a low LGE group (Fig. 2). We examined whether the parameters of liver disease were significantly different between the groups. However, there was no significant difference between the high and low LGE groups regarding MELD and Child-Pugh scores, nor single components of these composite scores (Table 3).

Since alcohol is regularly considered to impair cardiac function and alcoholic cardiomyopathy is a known complication of chronic alcohol consumption, we analyzed the association of the etiology of cirrhosis and the extent of LGE. In our cohort, a high LGE could be found in a significantly higher extent in the alcoholic cirrhosis group than in the non-alcoholic cirrhosis group (Table $4)$.

\section{Extent of LGE}

We analyzed whether the amount of LGE was associated with functional parameters of the cardiovascular system in the low and high LGE groups. There were no significant differences in stroke volume, ejection fraction, heart rate, and systolic and diastolic arterial blood pressure between these two groups (data not shown). Since hyperdynamic circulation to a varying degree was found in all patients in out study group, we examined the cardiac output, $\mathrm{CI}$ and presence or absence of hyperdynamic status defined as a Cardiac Index $(\mathrm{CI})$ of greater than or equal to $4 \mathrm{~L} / \mathrm{min} / \mathrm{m}^{2}$, or less than $4 \mathrm{~L} / \mathrm{min} / \mathrm{m}^{2}$, respectively. Cardiac output showed a trend $(\mathrm{p}=0.073)$ and CI ( $\mathrm{p}=0.014$, Fig. 4$)$ and hyperdynamic status $(p=0.029$, Table 5$)$ were significantly different between the low and high LGE groups. To evaluate established indicators for cirrhotic cardiomyopathy in our cohort we analyzed QTc-interval and signs of impaired diastolic function on echocardiography. Though QTc-interval was prolonged in $25 \%$ of the patients, E/A ratio by echocardiography was normal in all patients.

\section{Serological markers of cardiomyopathy}

NT-proBNP and hsTroponin $\mathrm{T}$ are established serological markers of ischemic and non-ischemic CMP and serum levels of these proteins were measured and these values were markedly increased in these patients (Table 6), while cTNT was within normal ranges.

We measured other biomarkers which have been associated with cardiomyopathy; of these, PLGF showed a significant correlation and sFLT1 showed a strong trend. Both were decreased in the high LGE group (Fig. 5).

\section{Discussion}

Liver failure in patients with ELD was the major life limiting factor until OLT has become the therapeutic option of choice resulting in a major improvement of survival. Subsequently, other comorbidities have become more apparent and clinically relevant. The increasing rates of cardiovascular complications after liver transplantation have raised the question of the underlying reasons for heart failure and electrical abnormalities. The increasing number of patients who receive liver transplantation has conferred a substantial body of evidence for hemodynamic deterioration following surgery. Accordingly, rates of pulmonary oedema have been reported to occur in up to $56 \%$ of liver transplant recipients following surgery, and hemodynamically significant arrhythmias in $27 \%$, and congestive heart failure in as many as 5.6\% [18]. In a more recent study, $82 / 179$ patients after OLT suffered from cardiac decompensation and cardiac causes were the leading cause of death in these patients [9]. Although

Table 2 Hemodynamic characteristics

\begin{tabular}{llll}
\hline Parameter/mean \pm SD & Cirrhosis patients & Controls & p \\
\hline Late enhancement [\%] & $27 \pm 16.5(2-62)$ & N/A & N/A \\
\hline Cardiac index [L/min/m²] & $4.36 \pm 0.86(3.41-6.48)$ & $3.6 \pm 0.74(2.10-6.31)$ & $<0.01$ \\
\hline Ejection fraction [\%] & $72 \pm 7.5(49-83)$ & $66 \pm 7.0(33-84)$ & $<0.01$ \\
\hline Stroke volume $[\mathrm{mL}]$ & $107 \pm 20.8(81-154)$ & $97.4 \pm 20.5(53-155)$ & 0.08 \\
\hline Cardiac output $[\mathrm{L} / \mathrm{min}]$ & $7.9 \pm 1.63(4.4-11.1)$ & $6.7 \pm 1.64(3.5-12.5)$ & $<0.01$ \\
\hline Heart rate at rest $[\mathrm{bpm}]$ & $74.9 \pm 11.9(50-104)$ & $64.8 \pm 9.4(45-91)$ & $<0.01$ \\
\hline Syst. blood pressure rest $[\mathrm{mmHg}]$ & $109.8 \pm 14.4(92-132)$ & $126.4 \pm 11.5(105-160)$ & $<0.01$ \\
\hline Diast. blood pressure rest $[\mathrm{mmHg}]$ & $61.2 \pm 7.8(44-76)$ & $76.4 \pm 9.0(46-104)$ & $<0.01$ \\
\hline LVEDV $[\mathrm{mL}]$ & $150.9 \pm 36.4(98-221)$ & $149.4 \pm 33.8(87-252)$ & 0.91 \\
\hline
\end{tabular}

LVEDV left ventricular enddiastolic volume; Range is in brackets; Mann-Whitney-U test for differences was performed 

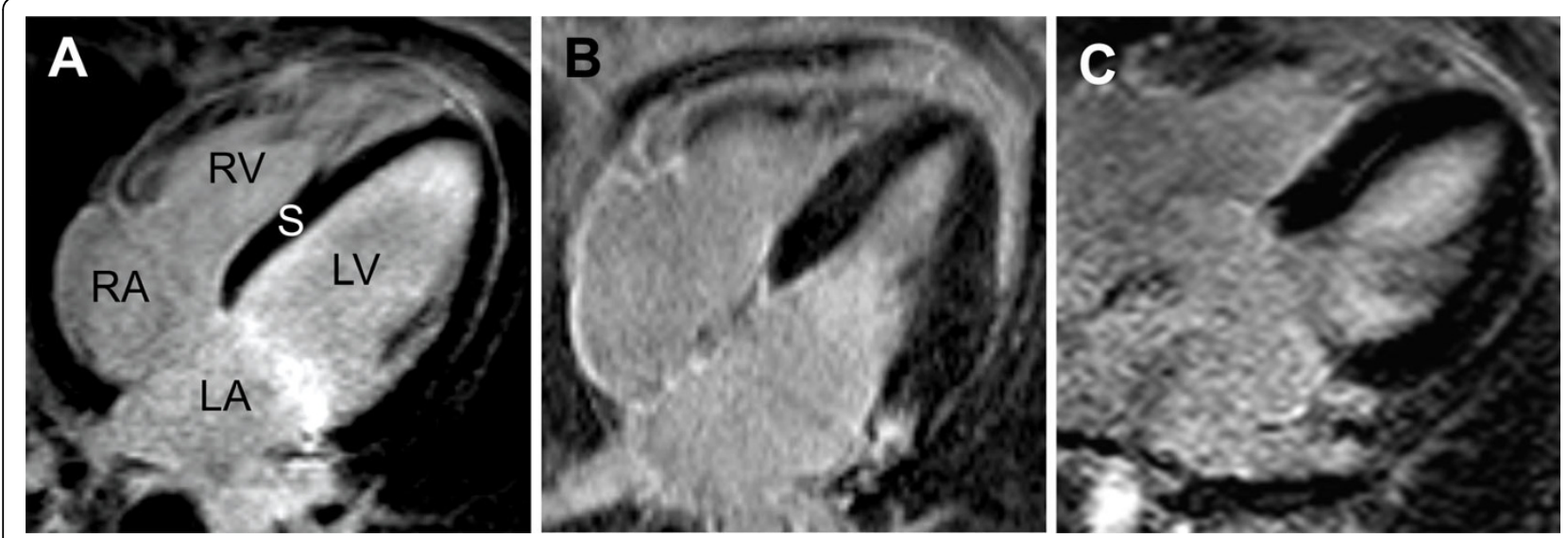

Figure 3 LGE imaging in a normal subject who showed no signs of late enhancement in a four-chamber view (A). Patient suffering from ELD. Patchy pattern of delayed contrast enhancement ( $B$ and $C$ ). $L A=$ left atrium, $R A=$ right atrium, $L V=$ left ventricle, $R V=$ right ventricle, $S=$ interventricular septum

adverse intraoperative cardiac events and history of cardiac disease are independent predictors of death due to cardiac cause, established risk assessment-including ECG, echocardiography, coronary angiography, and myocardial perfusion scintigraphy-fails to predict a complicated perioperative course or death attributed to cardiovascular events. A good diagnostic means for investigating cardiocirculatory changes before OLT is still lacking. For this reason, better detection of patients at risk for cirrhotic cardiomyopathy is required.

We therefore used CMR as the gold standard for the imaging of cardiomyopathies and biomarkers as wellestablished tools for the detection of heart failure. In line with previous findings, all patients in our study group showed a hyperdynamic cardiac function [4] (i.e. increased heart rate and cardiac output at rest suggesting healthy hearts). In contrast, NT-proBNP and hsTNT were elevated in those patients, indicating a concealed

Table 3 Parameters of liver disease

\begin{tabular}{|c|c|c|c|}
\hline Parameter & Low LGE & $\begin{array}{l}\text { High } \\
\text { LGE }\end{array}$ & $\begin{array}{l}\text { p (Mann-Whitney } \\
\text { U) }\end{array}$ \\
\hline MELD (mean \pm SD) & $\begin{array}{l}15.7 \pm \\
5.2\end{array}$ & $\begin{array}{l}12.3 \pm \\
4.7\end{array}$ & 0.15 \\
\hline $\begin{array}{l}\text { Child-Pugh Score (median; } \\
\text { range) }\end{array}$ & 10; $(7-11)$ & $8 ;(5-11)$ & 0.12 \\
\hline $\begin{array}{l}\text { Bilirubin (mean } \pm \text { SD) [mg/ } \\
\mathrm{dL}]\end{array}$ & $4.4 \pm 5.3$ & $2.1 \pm 1.4$ & 0.40 \\
\hline $\begin{array}{l}\text { Creatinine (mean } \pm \text { SD) [mg/ } \\
\mathrm{dL}]\end{array}$ & $1.0 \pm 0.5$ & $1.1 \pm 0.6$ & 0.87 \\
\hline INR (mean $\pm \mathrm{SD}$ ) & $\begin{array}{l}1.46 \pm \\
0.38\end{array}$ & $\begin{array}{l}1.15 \pm \\
0.16\end{array}$ & 0.06 \\
\hline Age (mean \pm SD) [years] & $\begin{array}{l}50.9 \pm \\
14.0\end{array}$ & $\begin{array}{l}53.3 \pm \\
6.0\end{array}$ & 1.00 \\
\hline
\end{tabular}

MELD score Model for End-Stage Liver Disease, INR International normalized ratio for Quick value; Range is in brackets, SD standard deviation form of heart failure. One could hypothesize that these markers are evidence of a barely compensated cardiocirculatory system close to the edge of decompensation.

Since hsTNT is supposed to uncover minimal damages of myocardial tissue [19], we performed LGE CMR to detect potential myocardial injury. One major finding in this study is that CMR allows detection of LGE in patients with end-stage liver disease.

LGE was traditionally only considered to be associated with fibrosis due to ischemic heart disease [11] but was then also described in non-ischemic cardiomyopathies, in infiltrative disorders such as amyloidosis and Fabry's disease [12], More recent reports have suggested there is enhancement in regions of myocarditis [20]. LGE can therefore be caused by fibrosis or pathological deposits in the myocardium as well as an inflammatory process. Recent work could show that the pattern of LGE is indeed characteristic of different cardiac diseases, allowing for an even better diagnosis and etiological allocation $[21,22]$.

Though LGE could be detected in patients regardless of etiology of liver disease, indicating a common mechanism originating from cirrhosis, the extent of LGE was more pronounced in patients with alcoholic liver cirrhosis. This supports the hypothesis of an additional modifier originating from alcohol abuse. The patchy pattern of LGE in our study group is comparable to the pattern found in acute myocarditis, where a partial reversibility of LGE in the chronic phase was described

\section{Table 4 LGE vs alcoholic etiology}

\begin{tabular}{lll}
\hline $\mathbf{N}$ & Non-alcoholic cirrhosis & Alcoholic cirrhosis \\
\hline Low LGE & 5 & 2 \\
\hline High LGE & 1 & 7 \\
\hline
\end{tabular}

$P=0.041$ (Fisher Exact Test), LGE late gadolinium enhancement 


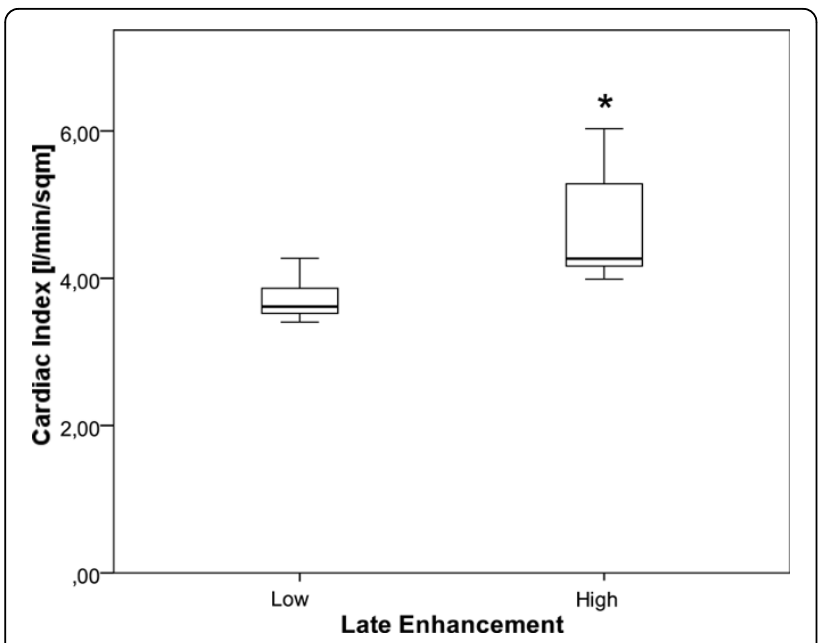

Figure 4 Box plots of $\mathrm{Cl}$ data are shown. The more pronounced hyperdynamic circulation in the high LGE group (right) compared to the low LGE group is depicted ( $p=0.029)$.

before [23]. However, patients with acute myocarditis show markedly elevated levels of conventional cTNT, which could not be detected in our cohort. The pattern differs from other non-ischemic CMP or after myocardial infarction, as mentioned before. Interestingly, there is a striking mismatch between the degree of LGE and the degree of LV function. Although a mean of $27 \%$ of the myocardial mass was affected by LGE, only one patient disclosed depressed systolic LV function. This indicates that the detected LGE in this study may not be due to fibrosis, but rather caused by pathological deposits (e.g., accumulated cardiotoxic metabolites, oxygen supply/demand mismatch due to arteriovenous pulmonary shunts, inflammation, a yet unknown mechanism). This might be explained by the findings of previous studies, which showed that cardiomyocytes and the trabecular network of the myocardium are changed in cirrhotic patients [3]. Histological specimens to examine possible alterations were not available in our patients.

In order to analyze correlations of established clinical parameters with LGE, we dichotomized our patient group, one with low amounts of LGE and one with high amounts of LGE. This resulted in a cut-off value for high LGE of approx. 25\% affected myocardium. These two groups were then compared in regard to liver and cardiocirculatory functions. Interestingly, we could not find a significant difference in markers of liver function

Table 5 LGE vs CI

\begin{tabular}{lll}
\hline $\mathbf{N}$ & $\mathrm{Cl}<\mathbf{4 ~ L / m i n} / \mathbf{m}^{\mathbf{2}}$ & $\mathrm{Cl} \geq \mathbf{4 ~ L / m i n} / \mathrm{m}^{\mathbf{2}}$ \\
\hline Low LGE & 6 & 1 \\
\hline High LGE & 1 & 6 \\
\hline
\end{tabular}

$\mathrm{P}=0.029$ (Fisher Exact Test), LGE late gadolinium enhancement
Table 6 Established and potential serological markers of cardiomyopathy

\begin{tabular}{llll}
\hline $\begin{array}{l}\text { Marker (mean } \pm \\
\text { SD) }\end{array}$ & Low LGE & High LGE & $\begin{array}{l}\text { P (Mann-Whitney } \\
\text { U) }\end{array}$ \\
\hline NT-proBNP [ng/L] & $\begin{array}{l}125.3 \pm \\
82.2\end{array}$ & $\begin{array}{l}101.8 \pm \\
64.1\end{array}$ & 0.18 \\
\hline hs-TNT [ng/L] & $5.6 \pm 4.2$ & $7.4 \pm 3.8$ & 0.56 \\
\hline SFLT1 [ng/L] & $143.7 \pm$ & $77.1 \pm 21.2$ & 0.06 \\
& 22.6 & & \\
\hline PLGF [ng/mL] & $36.8 \pm 10.3$ & $20.6 \pm 2.2$ & 0.02
\end{tabular}

hsTNT high sensitivity cTNT, LGE late gadolinium enhancement, NT-proBNP Nterminal prohormone brain natiuretic peptide, hsTNT high sensitivity cTNT, sFLT1 soluble short form of vascular endothelial growth factor receptor-1, PLGF placental growth factor

including MELD and Child scores between these two groups. An obvious link between liver function and LGE could therefore not be recognized. Nevertheless, we could demonstrate a significant difference regarding the CI between the low and high LGE groups. Cirrhosis leads to portal hypertension of varying extents. Complications of portal hypertension include the development of portosystemic collaterals ending among others in varices bearing the risk for variceal bleeding. Furthermore, portal hypertension results in peripheral and splanchnic vasodilatation and compensatory hyperdynamic cardiac function, which can be measured by an increased CI. As noted above there is a striking mismatch between LGE and LV function. In this context it might appear even more surprising that an even higher cardiac function as measured by cardiac index is associated with higher degrees of LE. This is vice versa to published data on LGE in other patient cohorts [24]. One could speculate that these circumstances result in a high cardiac workload burden and an incipient cardiac decompensation. Another explanation might be that detection of LGE in cirrhosis patients is caused by different mechanisms. And finally one can not exclude that the inclusion and exclusion criteria into this study might have influenced these outcomes. This would be in line with previous studies regarding cardiocirculatory regulation and hypercirculatory status in cirrhotic patients showing findings that have not yet been well explained [3]. In our study we could not find an association between LGE and other cardiac tests to be related to cirrhotic cardiomyopathy. Prolonged QTc-interval and reduced E/A ratio did not correlate with LGE. In fact the former could only be found in $25 \%$ of patients and the latter was normal in all patients. This might be attributed to the fact, that the aim of this study was not to examine patients with overt cardiac alterations, but detect common changes found in not preselected patients.

Since performing CMR might not be feasible in all ELD patients or contraindicated, we investigated several 


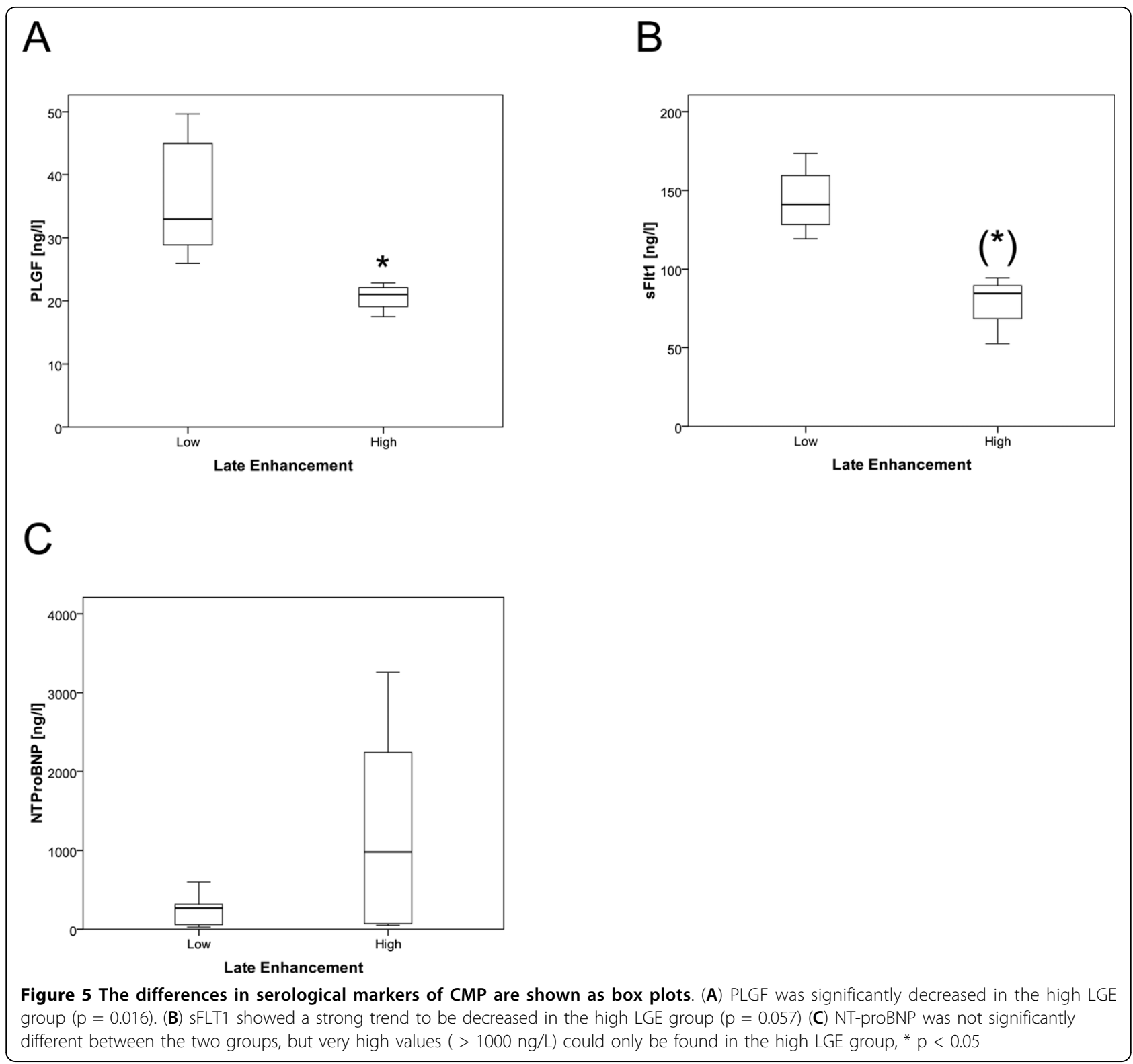

potential markers for cirrhotic cardiomyopathy. SFLT1 and PLGF were decreased in patients in the high LGE group. sFLT1 is the soluble short form of vascular endothelial growth factor receptor-1 (VEGFR-1), a member of the VEGFR family. It has been shown to interact with placental growth factor (PLGF). The involvement of both proteins in angiogenesis, the circulatory and renal dysregulation in preeclamptic patients, and more recently as part of the development of atherosclerosis have all been discussed [25], [26]. Most interestingly, VEGF, its receptors and PLGF have been implied in the regulation of neovascularization in portal hypertension. This implication not only points to new diagnostic but also to new therapeutic approaches [27],
[28]. Based on these data, one might speculate that these factors also play a role in circulatory dysregulation in ELD patients. Additionally, these biomarkers might be instrumental in detecting patients at risk and in monitoring them over time. No differences in other potential biomarkers were observed between the two groups.

How might these data affect clinical practice? Cardiac complications are a frequent cause of morbidity and mortality in patients undergoing liver transplantation and TIPS. These two interventions pose a stress on the cardiocirculatory system and can lead to a decompensation of cardiac function. This occurs despite regular and careful previous cardiological evaluation [9]. To our 
knowledge, this is the first description of LGE and hsTNT elevation in patients with ELD. CMR and hsTNT as well as NT-proBNP might enable clinicians to detect myocardial alterations, which is usually hampered by the hyperdynamic LV function in these patients. Further and larger studies are needed to confirm our results and in addition allow for more subtle subgroup analysis and focus on outcome data. These could then lead to a new risk stratification for cardiac complications based on these new diagnostic assessments and subsequently to an adapted and optimized perioperative management or to a treatment of the myocardial alterations itself. Furthermore it could lead to the exclusion of patients from OLT or TIPS due to high risk of development of heart failure after these interventions.

The association of the degree of LGE and hyperdynamic status in portal hypertension that could be demonstrated warrants further investigation and might be the basis for future studies examining its pathophysiological backgrounds.

Since previous studies could demonstrate a normalization of the hemodynamic parameters after liver transplantation in ELD patients [29], further studies have to be done, including follow-up measurements investigating the extent of a possible reversibility of these myocardial alterations.

\section{Conclusions}

Based on our findings, we conclude that myocardial alterations have a high prevalence among ELD patients, can be superbly detected and quantified by CMR and are linked to portal hypertension. Their exact role related to "cirrhotic cardiomyopathy" has to be determined. Due to its unique ability to provide non-invasive, highly sensitive tissue characterization combined with functional analysis, CMR may become the gold standard for the diagnosis of myocardial alterations and its severity as it is in other cardiac diseases. Future experimental and clinical work including outcome analysis is needed to clarify the mechanism and possible consequences for risk stratification and treatment, especially regarding patients undergoing TIPS or liver transplantation.

\footnotetext{
Abbreviations

Cl: Cardiac Index; CMP: Cardiomyopathy; CMR: cardiovascular magnetic resonance; CTNT: cardiac Troponin T; ELD: end-stage liver disease; FFE: Fast Field Echo; HSTNT: high sensitivity CTNT; INR: International normalized ratio for Quick value; LGE: late gadolinium enhancement; MELD: Model for EndStage Liver Disease; NT-PROBNP: N-terminal prohormone brain natiuretic peptide; OLT: orthotopic liver transplantation; PLGF: placental growth factor; SAX: short axis; SENSE: sensitivity encoding; SFLT1: soluble short form of vascular endothelial growth factor receptor-1; TE: Echo time; TIPS: transjugular intrahepatic portosystemic shunt; TR: Repetition time
}

\section{Acknowledgements}

We would like to thank Angela Wochele and Birgit Hoerig for excellent technical support.

\section{Grant support}

D.N.G. was supported by the PostDoc Fellowship program of the University of Heidelberg.

\section{Author details}

'Department of Internal Medicine III, University Hospital of Heidelberg, Im Neuenheimer Feld 410, 69120 Heidelberg, Germany. ${ }^{2}$ Department of Internal Medicine IV, University Hospital of Heidelberg, Im Neuenheimer Feld 410, 69120 Heidelberg, Germany.

\section{Authors' contributions}

DL study design, data acquisition and analysis, writing the manuscript. HS data analysis and writing the manuscript. AZ data acquisition and analysis, SL data acquisition, CW data acquisition and analysis, KHW data analysis, EG study design and writing the manuscript, WS study design and data analysis, PS data acquisition and analysis, HAK study design and writing the manuscript, DNG study design, data acquisition and analysis and writing the manuscript. All authors approved the final version of the manuscript.

\section{Competing interests}

E. Giannitsis and $\mathrm{H}$. Katus have served as speakers for and received grants from Roche Diagnostics. H. Katus holds patents jointly for CTNT assay and hsTNT assay.

Received: 11 March 2010 Accepted: 13 August 2010

Published: 13 August 2010

\section{References}

1. Cardenas A, Gines P: Portal hypertension. Curr Opin Gastroenterol 2009, 25(3):195-201.

2. Menon KV, Kamath PS: Regional and systemic hemodynamic disturbances in cirrhosis. Clin Liver Dis 2001, 5(3):617-627, viii.

3. Moller $\mathrm{S}$, Henriksen JH: Cardiovascular complications of cirrhosis. Gut 2008, 57(2):268-278.

4. Lee RF, Glenn TK, Lee SS: Cardiac dysfunction in cirrhosis. Best Pract Res Clin Gastroenterol 2007, 21(1):125-140.

5. Ocel JJ, Edwards WD, Tazelaar HD, Petrovic LM, Edwards BS, Kamath PS: Heart and liver disease in 32 patients undergoing biopsy of both organs, with implications for heart or liver transplantation. Mayo Clin Proc 2004, 79(4):492-501.

6. Alqahtani SA, Fouad TR, Lee SS: Cirrhotic cardiomyopathy. Semin Liver Dis 2008, 28(1):59-69.

7. Rosado B, Kamath PS: Transjugular intrahepatic portosystemic shunts: an update. Liver Transpl 2003, 9(3):207-217.

8. Ripoll C, Catalina MV, Yotti R, Olmedilla L, Perez-Pena J, Lo lacono O, Rincon D, Garcia-Fernandez MA, Bermejo J, Banares R: Cardiac dysfunction during liver transplantation: incidence and preoperative predictors. Transplantation 2008, 85(12):1766-1772.

9. Fouad TR, Abdel-Razek WM, Burak KW, Bain VG, Lee SS: Prediction of cardiac complications after liver transplantation. Transplantation 2009, 87(5):763-770.

10. Pattynama PM, Lamb HJ, van der Velde EA, van der Wall EE, de Roos A: Left ventricular measurements with cine and spin-echo MR imaging: a study of reproducibility with variance component analysis. Radiology 1993, 187(1):261-268.

11. Kim RJ, Wu E, Rafael A, Chen EL, Parker MA, Simonetti O, Klocke FJ, Bonow RO, Judd RM: The use of contrast-enhanced magnetic resonance imaging to identify reversible myocardial dysfunction. N Engl J Med 2000 343(20):1445-1453.

12. Vogel-Claussen J, Rochitte CE, Wu KC, Kamel IR, Foo TK, Lima JA, Bluemke DA: Delayed enhancement MR imaging: utility in myocardial assessment. Radiographics 2006, 26(3):795-810. 
13. Teraoka K, Hirano M, Ookubo H, Sasaki K, Katsuyama H, Amino M, Abe Y, Yamashina A: Delayed contrast enhancement of MRI in hypertrophic cardiomyopathy. Magn Reson Imaging 2004, 22(2):155-161.

14. Bandettini WP, Arai AE: Advances in clinical applications of cardiovascular magnetic resonance imaging. Heart 2008, 94(11):1485-1495.

15. Cerqueira MD, Weissman NJ, Dilsizian V, Jacobs AK, Kaul S, Laskey WK, Pennell DJ, Rumberger JA, Ryan T, Verani MS: Standardized myocardial segmentation and nomenclature for tomographic imaging of the heart: a statement for healthcare professionals from the Cardiac Imaging Committee of the Council on Clinical Cardiology of the American Heart Association. Circulation 2002, 105(4):539-542.

16. Cheng AS, Robson MD, Neubauer S, Selvanayagam JB: Irreversible myocardial injury: assessment with cardiovascular delayed-enhancement MR imaging and comparison of 1.5 and 3.0 T-initial experience. Radiology 2007, 242(3):735-742.

17. Friedrich MG: Tissue characterization of acute myocardial infarction and myocarditis by cardiac magnetic resonance. JACC Cardiovasc Imaging 2008, 1(5):652-662.

18. Donovan CL, Marcovitz PA, Punch JD, Bach DS, Brown KA, Lucey MR, Armstrong WF: Two-dimensional and dobutamine stress echocardiography in the preoperative assessment of patients with endstage liver disease prior to orthotopic liver transplantation. Transplantation 1996, 61(8):1180-1188.

19. Katus HA, Giannitsis $E$, Jaffe $A S$, Thygesen K: Higher sensitivity troponin assays: Quo vadis? Eur Heart J 2009, 30(2):127-128

20. Kim DH, Choi SI, Chang HJ, Choi DJ, Lim C, Park JH: Delayed hyperenhancement by contrast-enhanced magnetic resonance imaging: Clinical application for various cardiac diseases. J Comput Assist Tomogr 2006, 30(2):226-232.

21. Cummings KW, Bhalla S, Javidan-Nejad C, Bierhals AJ, Gutierrez FR, Woodard PK: A pattern-based approach to assessment of delayed enhancement in nonischemic cardiomyopathy at MR imaging. Radiographics 2009, 29(1):89-103.

22. Bohl S, Wassmuth R, Abdel-Aty H, Rudolph A, Messroghli D, Dietz R, SchulzMenger J: Delayed enhancement cardiac magnetic resonance imaging reveals typical patterns of myocardial injury in patients with various forms of non-ischemic heart disease. Int I Cardiovasc Imaging 2008, 24(6):597-607.

23. Goitein O, Matetzky S, Beinart R, Di Segni E, Hod H, Bentancur A, Konen E: Acute myocarditis: noninvasive evaluation with cardiac MRI and transthoracic echocardiography. AJR Am J Roentgenol 2009, 192(1):254-258.

24. Beek AM, Bondarenko O, Afsharzada F, van Rossum AC: Quantification of late gadolinium enhanced CMR in viability assessment in chronic ischemic heart disease: a comparison to functional outcome. $J$ Cardiovasc Magn Reson 2009, 11:6.

25. Shibuya M: Vascular endothelial growth factor receptor-1 (VEGFR-1/Flt-1): a dual regulator for angiogenesis. Angiogenesis 2006, 9(4):225-230, discussion 231.

26. Mutter WP, Karumanchi SA: Molecular mechanisms of preeclampsia. Microvasc Res 2008, 75(1):1-8.

27. Fernandez M, Mejias M, Garcia-Pras E, Mendez R, Garcia-Pagan JC, Bosch J: Reversal of portal hypertension and hyperdynamic splanchnic circulation by combined vascular endothelial growth factor and plateletderived growth factor blockade in rats. Hepatology 2007, 46(4):1208-1217.

28. Van Steenkiste C, Geerts A, Vanheule E, Van Vlierberghe H, De Vos F, Olievier K, Casteleyn C, Laukens D, De Vos M, Stassen JM, Carmeliet P, Colle I: Role of Placental Growth Factor in Mesenteric Neo-Angiogenesis in a Mouse Model of Portal Hypertension. Gastroenterology 2009, 137:2112-24

29. Torregrosa M, Aguade S, Dos L, Segura R, Gonzalez A, Evangelista A, Castell J, Margarit C, Esteban R, Guardia J, Genescà J: Cardiac alterations in cirrhosis: reversibility after liver transplantation. J Hepatol 2005, 42(1):68-74

doi:10.1186/1532-429X-12-47

Cite this article as: Lossnitzer et al: Myocardial late gadolinium enhancement cardiovascular magnetic resonance in patients with cirrhosis. Journal of Cardiovascular Magnetic Resonance 2010 12:47.

\section{Submit your next manuscript to BioMed Central and take full advantage of:}

- Convenient online submission

- Thorough peer review

- No space constraints or color figure charges

- Immediate publication on acceptance

- Inclusion in PubMed, CAS, Scopus and Google Scholar

- Research which is freely available for redistribution

Submit your manuscript at www.biomedcentral.com/submit
Biomed Central 